Case Report

\title{
An Autistic Female with Refractory Juvenile Myoclonic Epilepsy and Sequelae of Neonatal Cerebellar Insults, Effectively Treated with Perampanel
}

\author{
Atushi Nishimura ${ }^{1,2}$, Toshiaki Abe ${ }^{1,3, *}$, Takao Harasawa ${ }^{1}$, Hiroshi Oba ${ }^{4}$ \\ ${ }^{1}$ Department of Pediatric Neurology, Ashikaganomori Ashikaga Hospital, Tochigi, Japan \\ ${ }^{2}$ Department of Pediatrics, Nihon University School of Medicine, Tokyo, Japan \\ ${ }^{3}$ Department of Pediatrics, Teikyo University School of Medicine, Tokyo, Japan \\ ${ }^{4}$ Department of Radiology, Teikyo University School of Medicine, Tokyo, Japan
}

Email address:

ganjigansan $a$ gmail.com (T. Abe)

${ }^{*}$ Corresponding author

To cite this article:

Atushi Nishimura, Toshiaki Abe, Takao Harasawa, Hiroshi Oba. An Autistic Female with Refractory Juvenile Myoclonic Epilepsy and Sequelae of Neonatal Cerebellar Insults, Effectively Treated with Perampanel. American Journal of Psychiatry and Neuroscience. Vol. 6, No. 3, 2018, pp. 72-77. doi: 10.11648/j.ajpn.20180603.14

Received: May 31, 2018; Accepted: August 6, 2018; Published: September 3, 2018

\begin{abstract}
The patient was a 27-year-old mildly intellectually disabled woman with autistic spectrum disorder (ASD) and juvenile myoclonic epilepsy (JME). The patient's condition had shown resistance to many antiepileptic drugs; however, perampanel (PER) was effective. The patient's electroencephalogram (EEG) records were consistent with those of JME, and brain magnetic resonance imaging showed a normal cerebrum and limbic system but the characteristic sequelae of cerebellar insults, such as cerebellitis. The discontinuation of lamotrigine (LTG) induced suspected myoclonic atonic seizures (MAS) that were worsened by adding levetiracetam (LEV) but improved by the administration of rafinamide (RFN). The further administration of PER improved all symptoms and EEG findings of the patient within two weeks, and the patient has been seizure-free for more than two years. The present case report demonstrates that the sequelae of neonatal cerebellitis can cause JME, and the withdrawal of LTG induces myoclonic atonic seizures. PER can modify the pathogenic cerebellar focus of JME.
\end{abstract}

Keywords: Juvenile Myoclonic Epilepsy, Cerebellar Hyperintensity Area, Sequel, Cerebellar Insults, Cerebellar Seizures, Lamotrigine Withdrawal Syndrome, Perampanel

\section{Introduction}

JME is categorized as an idiopathic generalized epilepsy, characterized by generalized tonic clonic seizures (GTS), myoclonic seizures (MS), absence seizures (AS), without myoclonic atonic seizures (MAS). In most cases, the brain magnetic resonance imaging (MRI) findings are normal. Interictal electroencephalography (EEG) shows $3-$ to $6-\mathrm{Hz}$ generalized spikes or polyspike-wave discharges. While JME is commonly treated with valproate, the condition is intractable in patients with psychoses such as ASD [1-2]. The epileptogenesis of JME in the cerebrum has been studied [3-5], and the involvement of the cerebellum has been reported [6-10]. However, the etiologies remain to be determined, as JME is thought to be a spectrum disorder rather than a collection of diseases [11].

\section{Case Presentation}

The present patient was hospitalized as a neonate due to suspected cerebellitis. The patient developed motor clumsiness and was diagnosed with ASD and mild intellectual disability based on behavior. The patient experienced febrile seizures at two years of age and GTS from seven years of age; these conditions were effectively treated with carbamazepine (CBZ). The patient suffered myoclonic status epilepticus at 15 
years of age and was admitted to a hospital and diagnosed with JME by a neurologist, based on the presence of EEG findings characteristic of JME. The patient was treated with valproic acid (VPA) and clonazepam (CZP), which showed partial efficacy. The patient visited the outpatient clinic of author's hospital with a chief complaint of refractory JME at 19 years of age, showing abnormal MRI findings attributed to the sequelae of neonatal cerebellitis and abnormal EEG findings (Figure 1, Figure 2).

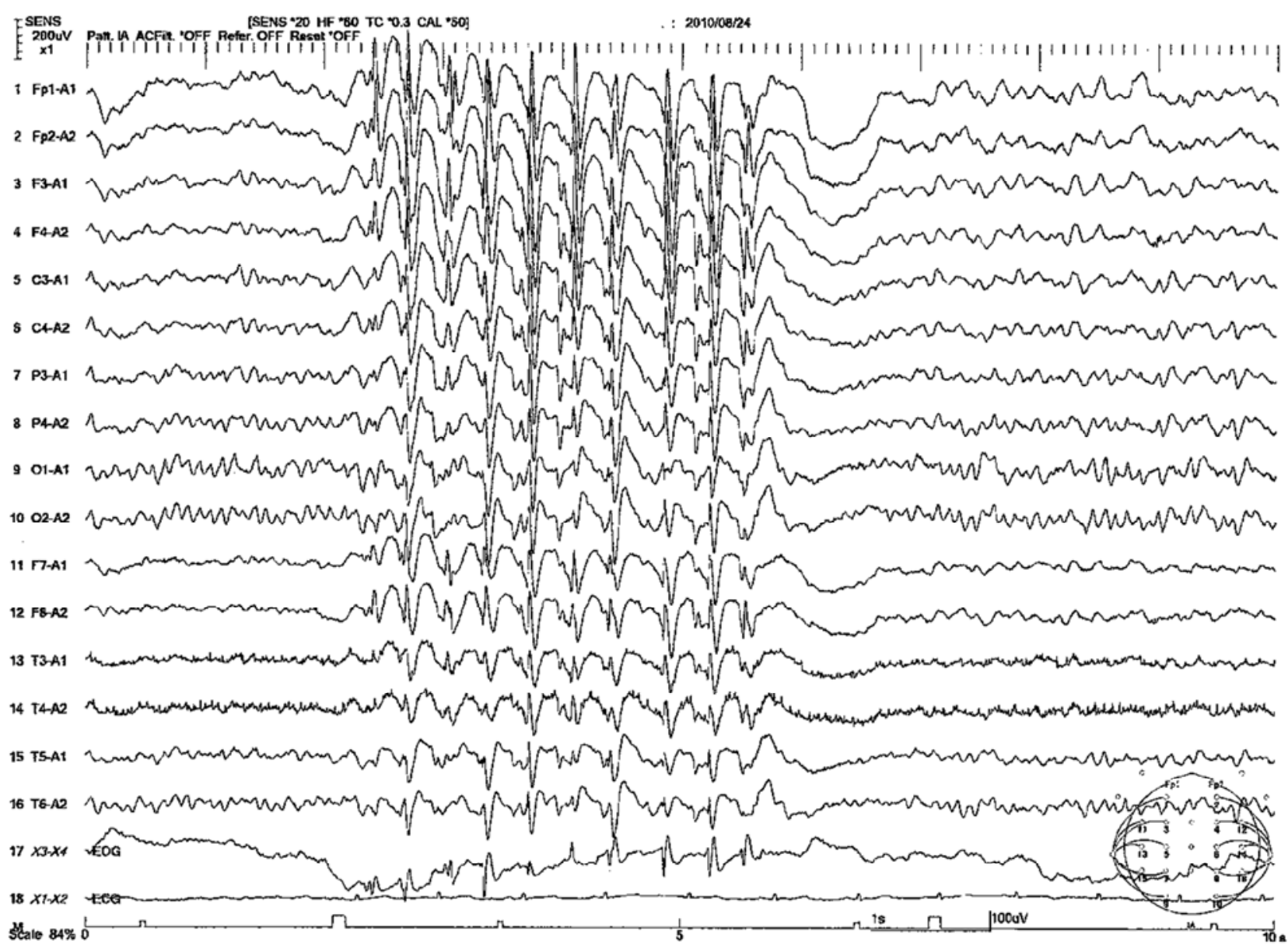

Figure 1. The interictal EEG recording (8,2010) of the patient with JME (MS, absence seizure and GTC) treated by VPA (600mg) showed frequent paroxysmal discharge of 3-6Hz spike and wave, polyspikes and wave complex, and sporadically bilateral independent frontal spike and wave [10].

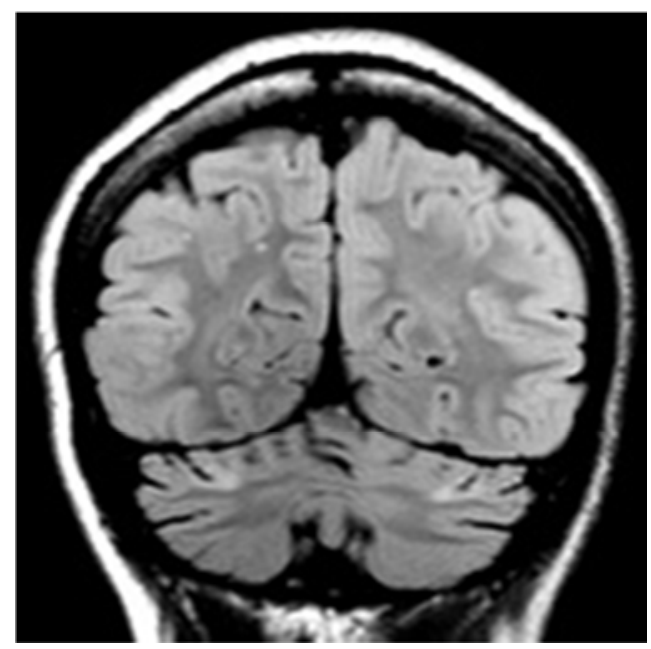

Figure 2. Brain MRI. There was no cerebral atrophy. Slight atrophy was observed in the cerebellar hemisphere and vermis. Hyperintense areas were observed symmetrically in the cerebellar hemispheres surrounding the horizon fissures on T2-weighted and FLAIR image. Non-specific, small hyperintense spots were seen in the cerebral white matter on FLAIR and T2-weighted axial, coronal and sagittal images. These images showed no abnormalities in the cerebral gray or white matter, or in the limbic system, however destruction was apparent in both atrophic cerebellar hemispheres and the vermis. These sequelae of old insults in the cerebellum are suggestive of the dysfunction of cerebellum-thalamic circuit and the pathogenic epileptic focuses of JME or cerebellar seizures [10-11]. 
The patient was treated with VPA, CZP, lamotrigine (LTG), levetiracetam (LEV) and rafinamide (RFN), each of which showed side effects with no or only partial efficacy, as shown in Tableland Figure 3.

Table 1. The effects of various anticonvulsants on seizure symptoms of the patient.

\begin{tabular}{|c|c|c|c|c|c|c|c|c|c|}
\hline & Ms & Absence & Mas & Automatism & Gtes & \#Psychiatrics & Others & Concurrent (mg) & Failed \\
\hline CB Z (400) & $(+)$ & $(+)$ & & & $(-)$ & & & None & $\mathrm{CBZ}$ \\
\hline VPA (600) & $(+$ or- $)$ & $(-)$ & & & $(+$ or- $)$ & & & $\mathrm{CZP}(0.5), \mathrm{BZP}(5)$ & BZP \\
\hline CZP (1) & $(-)$ & $(-)$ & & & & & Stomatitis & VPA $(600)$ & $\mathrm{CZP}$ \\
\hline LTG (200) & $(+$ or- $)$ & & $(+$ or- $)$ & $(+)$ & & & severe rash & VPA $(800)$ & LTG \\
\hline $\operatorname{LEV}(1000)$ & $(+$ or -$)$ & $(+)$ & $(+)$ & $(+)$ & $(-)$ & & & VPA (1000), CZP (2) & LEV \\
\hline RFN (1200) & $(-)$ & $(+)$ & $(+$ or- $)$ & $(-)$ & $(+)$ & & & VPA (800), CNP (2) & \\
\hline PER (4) & $(-)$ & $(-)$ & $(-)$ & $(-)$ & $(-)$ & $(+)$ & & VPA (800), CZP (2), RFN (600) & \\
\hline
\end{tabular}

Notes: With gradual titration of each medication. Numbers in parentheses indicate mg.

CBZ; carbamazepin, VPA; valproic acid, CZP; chlonazepam, LTG; lamotrigine, LEV; levetiracetam, RFN; rafinamide, PER; perampanel, ms; myoclonic seizures, mas; myoclonic atonic seizures, gtc; generalized tonic clonic seizures, (-); improved, (+); worsened, \# psychiatric; infantification, violent behaviors

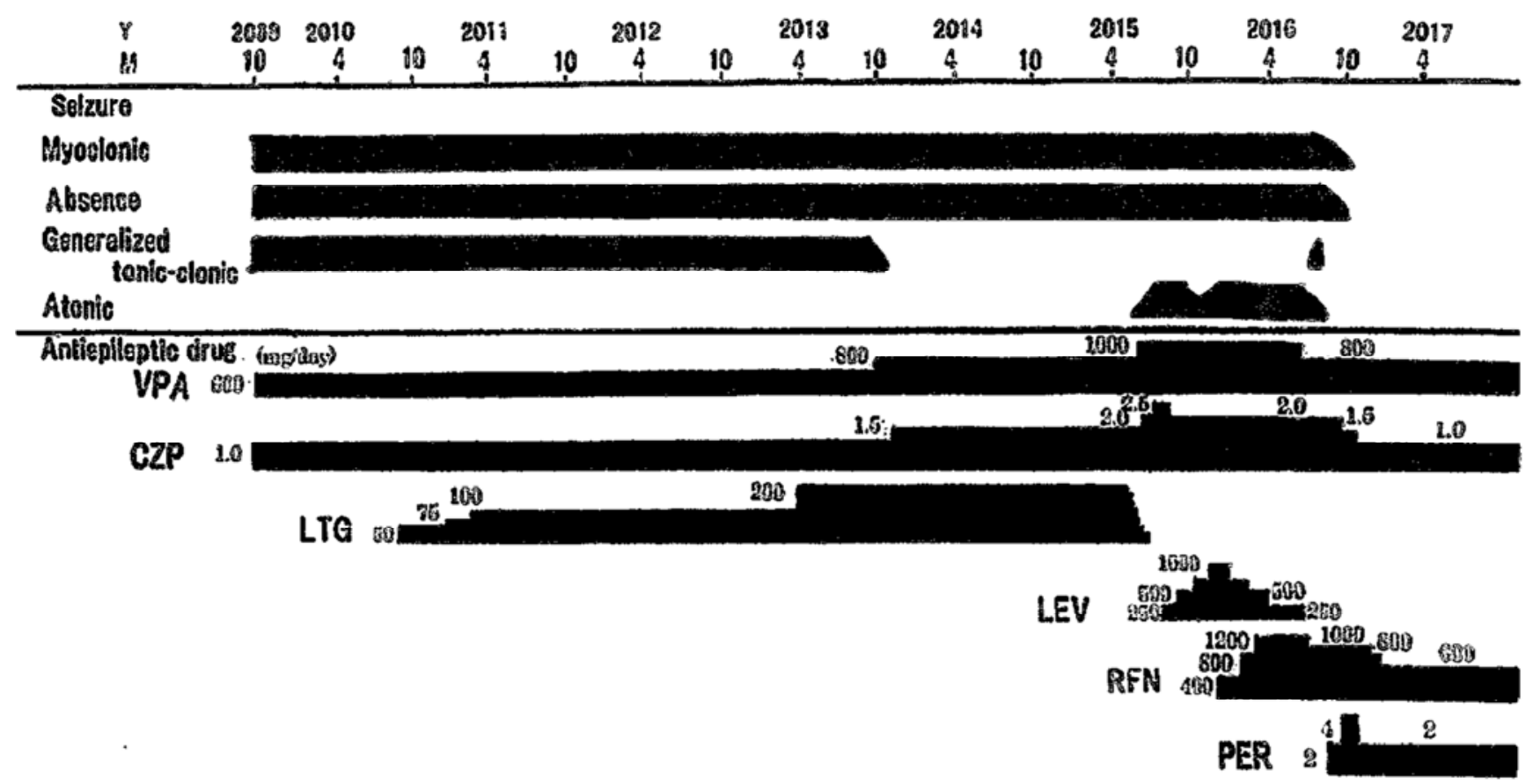

Figure 3. Seizures and the medication course chart.

LTG was discontinued due to side effects of severe oral rashes. As a result, the dose of LTG (200mg) was reduced by $25 \mathrm{mg}$ once every 3 days. The occurrence of suspected MASs was revealed one month after discontinuation of LTG. The seizure symptoms were myoclonic seizures followed by momentary atonic seizures several times every morning. When the patient experienced the symptoms for the first time, the patient and patient's mother quickly consulted the doctor to undergo the further examination (Figure 4) and treatment of patient's symptoms. 


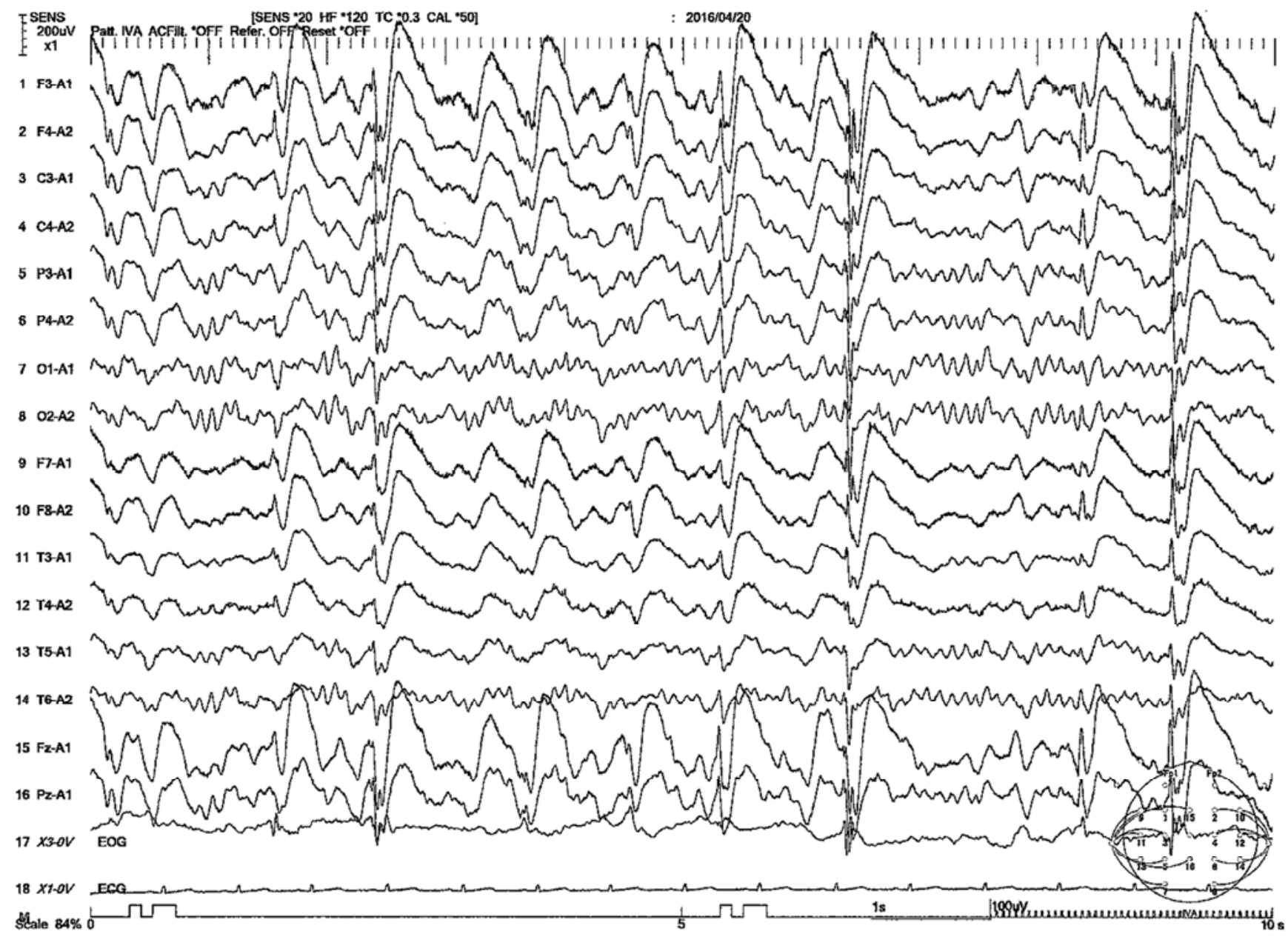

Figure 4. The interictal EEG recording (4,2016) from the patient who showed suspected MAS several times every morning under treatment with VPA (800mg), CZP (1mg), and LEV (250- to 1500-mg). The abnormal EEG recordings were induced by LTG and worsened by LEV. These EEG findings were similar to those from patients with MAS [19]. The awake, photoparoxysmal responses to 6- to 21-Hz intermittent photic stimulation provoked background slowing and bilateral synchronous 2-to3-Hz spike and waves burst.

The seizures worsened by the addition of $\geq 250 \mathrm{mg}$ of LEV and were improved by the replacement of LEV with $\geq 1000 \mathrm{mg}$ of RFN; however, the GTC and AS remained.

The addition of $2 \mathrm{mg}$ of PER to the concurrent drugs (CNP2 $\mathrm{mg}$, VPAR1000mg, RFN1000mg) improved both the patient's seizures and EEG findings (Figure 4) after 2 weeks. However, the patient showed violent and immature behavior with a dose of $4 \mathrm{mg}$; so thus, the dose was switched back to $2 \mathrm{mg}$. This reduced patient's abnormal behaviors, including patient's autistic behaviors. The patient has since remained seizure-free for more than two years.

\section{Discussion}

In neonates, the cerebellum is still developing and perinatal insults to the cerebellum may result in dysfunction of the tissue [14] and induce the overexpression of AMPA receptors on cerebellar neurons, which cause cerebellar seizures and myoclonic seizures, as are observed in Batten disease [15]. PER acts on the AMPA receptors of cerebellar neuronal cell membranes to decrease the excitable transmission and reduce the seizure activities [16-17]. This may be the mechanism by which the improvement of seizures was achieved in the present JME patient. The same mechanism can be used to explain for the improvement of the patient's autistic behavior [18].

The disease course of the patient is consistent with that of other JME patients; however, the patient showed abnormal EEG recordings (Figure 4) and myoclonic seizures followed by momentary atonic seizures several times every morning, and such symptoms were exacerbated by LEV and ameliorated by RFN. These symptoms are characteristic of myoclonic atonic epilepsy, which occurs in infant and young children, not in adults $[1,19]$. The mechanism by which these symptoms occurred in the present case is unclear but may involve either the manifestation of the original seizures or a withdrawal syndrome during the cessation of LTG [20-22]. To the best of the authors' knowledge, this is the first description of myoclonic atonic seizures in an adult JME case that were induced by LTG, worsened by LEV, and improved by RFN and PER. Further studies are needed to accumulate data on drug-related myoclonic atonic seizures in order to clarify the mechanism. 


\section{Conclusion}

An autistic woman with mild intellectually disability, refractory juvenile myoclonic epilepsy, and sequelae of neonatal cerebellar insults was effectively treated with PER.

MRI brain images from the present case showed no abnormal findings in the cerebral gray or white matter, or in the limbic system; however, destruction due to insults of the surrounding horizon fissures were apparent in both atrophic cerebellar hemispheres. These sequelae of old insults in the cerebellum may have been the lesions responsible for the disfunction of the cerebellum-thalamic circuit in JME or cerebellar seizures.

To the best of the authors' knowledge, this is the first description of myoclonic atonic seizures in an adult patient with JME case that was induced by LTG, worsened by LEV, and improved by RFN and PER. PER might be effective for controlling seizures and ameliorating the associated neuronal dysfunction in cases of refractory JME.

\section{Disclosure of Conflicts of Interest}

No authors have any conflicts of interest to disclose.

\section{Financial Disclosure/Funding}

The authors received no financial support for the research, authorship or publication of this article.

\section{Ethical Publication Statement}

The patient and her caregiver assented to the publication of this manuscript, which includes potentially identifying information.

We confirm that we have read the Journal's position on the issues involved in ethical publication and affirm that this report is consistent with those guidelines.

\section{References}

[1] Höfler J, Unterberger I, Dobesberger J, Kuchukhidze G, Walser G, Trinka E. Seizure outcome in 175 patients with juvenile myoclonic epilepsy-A long-term observational study. Epilepsy Research 2014; 108:1817-1824.

[2] Sansa G, Carlson C, Doyle W, Weiner HL, Bluvstein J, Barr W, Devinsky O. Medically refractory epilepsy in autism. Epilepsia 2011; 52:1071-1075. doi:1111/.1528-1167.2011. 03069.x.

[3] Jiang S, Luo C, Liu Z, Hou C, Wang P, Dong L, Zhong C, Lai Y, Xia Y, Yao D. MR spectroscopy shows reduced frontal lobe concentrations of $\mathrm{N}$-acetyl aspartate in patients with juvenile myoclonic epilepsy. Neural Plast 2016; 3547203. Epub 2015 Dec 28.

[4] Tae WS, Joo EY, Han SJ, Lee KH, Hong SB. CBF changes in drug naive juvenile myoclonic epilepsy patients. J Neurol 2007; 254:1073-80. Epub 2007 Mar 12.

[5] O'Muircheartaigh J ${ }^{1}$, Vollmar C, Barker GJ, Kumari V, Symms MR, Thompson P, Duncan JS, Koepp MJ, Richardson MP.
Abnormal thalamocortical structural and functional connectivity in juvenile myoclonic epilepsy. Brain. 2012; 135 (Pt 12):3635-44. doi: 10.1093/brain/aws296.

[6] Jiang S, Luo C, Gong J, Peng R, Ma S, Tan S, Ye G, Dong L, Yao D. Aberrant Thalamocortical Connectivity in Juvenile Myoclonic Epilepsy. Int J Neural Syst. 2017; 13:1750034. doi: 10.1142/S0129065717500344. [Epub ahead of print].

[7] Kotini $A^{1}$, Mavraki E, Anninos P, Piperidou H, Prassopoulos P Magnetoencephalographic findings in two cases of juvenile myoclonus epilepsy. Brain Topogr 2010; 23:41-5. doi: 10.1007/s10548-009-0114-5. Epub 2009 Sep 29.

[8] Kano M, Hashimoto K, Tabata T. Type-1 metabotropic glutamate receptor in cerebellar Purkinje cells: a key molecule responsible for long-term depression, endocannabinoid signalling and synapse elimination. Philos Trans R Soc Lond B Biol Sci 2008; 363 (1500):2173-2186.

[9] Benagiano V, Rizzi A, Lorusso L, Flace P, Saccia M, Cagiano R, Ribatti D, Roncali L, Ambrosi G. The functional anatomy of the cerebrocerebellar circuit: A review and new concepts. J Comp Neurol 2017; 14: doi: 10.1002/cne.24361. [Epub ahead of print].

[10] Boop S, Wheless J, Van Poppel K, McGregor A, Boop FA. Cerebellar seizures. J Neurosurg Pediatr 2013; 12:288-92. doi: 10.3171/2013.5. PEDS1394. Epub 2013 Jun 28.

[11] Baykan B, Wolf P. Juvenile myoclonic epilepsy as a spectrum disorder: A focused review. Seizure 2017; 49:36-41. doi: 10.1016/j.seizure.2017.05.011. Epub 2017 May 18.

[12] De Bruecker Y, Claus F, Ballaux F, et al. MRI findings in acute cerebellitis. European Radiology 2004; 14:1478-1483.

[13] Al-Maawali A, Blaser S, Zhao XY, Yoon G. Prospective study of activities of daily living outcomes in children with cerebellar atrophy. Dev Med Child Neurol 2014; 56:460-7. doi: 10.1111/dmcn.12289. Epub 2013 Oct 1.

[14] Annegers JF, Coan SP, Hauser WA, Leestma J. A population-based study of seizures after traumatic brain injuries. N Engl J Med 1998; 338:20-24.

[15] Kovács AD, Hof C, Pearce DA. Abnormally increased surface expression of AMPA receptors in the cerebellum, cortex and striatum of $C \ln 3$ (-/-) mice. Neurosci Lett 2015; 607:29-34. doi: 10.1016/j.neulet.2015.09.012. Epub 2015 Sep 12.

[16] Rogawski MA, Hanada T. Preclinical pharmacology of perampanel, a selective non-competitive AMPA receptor antagonist. Acta Neurol Scand Suppl 2013; 197:19-24. doi: 10.1111/ane.12100.

[17] Rogawski MA. AMPA receptors as a molecular target in epilepsy therapy. Acta Neurol Scand Suppl 2013; 197:9-18. doi: 10.1111/ane.12099.

[18] Lippman-Bell JJ, Rakhade SN, Klein PM, Obeid M, Jackson MC, Joseph A, Jensen FE. AMPA receptor antagonist NBQX attenuates later-life epileptic seizures and autistic-like social deficits following neonatal seizures. Epilepsia 2013; 54:1922-32. doi: 10.1111/epi.12378.

[19] Kelly SA, Kossoff EH. Doose syndrome (myoclonic-astatic epilepsy): 40 years of progress. Devlop Med \& Child Neurol 2010; 52:988-993.

[20] Wang-Tilz Y, Tilz C, Wang B, Pauli E, Koebnick C, Stefan H. Changes of seizures activity during rapid withdrawal of lamotrigine. Eur J Neurol 2005; 12:280-8. 
[21] Kröll-Seger J1, Mothersill IW, Novak S, Sälke-Kellermann RA, Krämer G. Levetiracetam-induced myoclonic status epilepticus in myoclonic-astatic epilepsy: a case report. Epileptic Disord 2006; 8:213-8.

[22] Albini M, Morano A, Fanella M, Lapenta L, Casciato S,
Fattouch J, Manfredi M, Giallonardo AT, Di Bonaventura C. Effectiveness of rufinamide in the treatment of idiopathic generalized epilepsy with atypical evolution: case report and review of the literature. Clin EEG Neurosci 2016; 47:162-6. doi: 10.1177/1550059414559940. Epub 2014 Nov 23. 